\title{
Coherent and incoherent production of vector mesons in ultraperipheral collisions of xenon ions within the QCD parton saturation approach
}

\author{
F. Kopp and M. V. T. Machado \\ High Energy Physics Phenomenology Group, GFPAE IF-UFRGS, \\ Caixa Postal 15051, CEP 91501-970, Porto Alegre, RS, Brazil
}

(Received 30 May 2018; published 9 July 2018)

\begin{abstract}
In this paper, we analyze the exclusive vector meson photoproduction in the recent run using xenon ions at energy of $5.44 \mathrm{TeV}$ performed by the Large Hadron Collider. We focus on the ultraperipheral collisions and provide theoretical predictions for coherent and incoherent cross sections within the color dipole approach and gluon saturation framework. The rapidity distribution is investigated in both cases and comparison to other approaches available in the literature is completed. We show that the expected yields are enough to perform reliable cross section measurements for light mesons as $\rho^{0}$ and $\phi$.
\end{abstract}

DOI: 10.1103/PhysRevD.98.014010

\section{INTRODUCTION}

Investigating the exclusive meson photoproduction in ultraperipheral collisons (UPCs) [1] is an essential tool to understand the underlying dynamics of strong interactions. In the case of heavy meson production, it sheds light in the low-x physics and helps to constrain the nuclear gluon density especially at large meson rapidities, $y$. As an example, in the $J / \psi$ photonuclear production at the Large Hadron Collider (LHC) regime, one obtains the value $x=\frac{m_{\psi}}{\sqrt{s_{A A}}} e^{-y} \simeq 3 \times 10^{-5}$ at $y=3$ for $\mathrm{PbPb}$ collisions. In the UPCs, the nuclear target is probed by quasivirtual photons, and the typical momentum scale is the meson mass. For light mesons, the mass scale lies below $1 \mathrm{GeV}$ and usual methods of weak coupling are not valid. In addition, even for the heavy meson production, the mass value is typically associated to a semihard scale below $10 \mathrm{GeV}$. These features make the theoretical approaches considering parton saturation very appealing. The reason is that the typical momentum scale at very low $x$ would be the nuclear saturation scale, $Q_{\text {sat }, A}^{2} \simeq A^{1 / 3} Q_{\text {sat }, p}^{2} \sim 6 \mathrm{GeV}^{2}$ (for $\mathrm{Pb}$ at very low- $x$ ), which is enhanced in the case of large nuclei. A demonstration of the power of predictability of the parton saturation approach including the geometric scaling phenomenon has been presented in Ref. [2], where the exclusive production of $\rho$ and $J / \psi$ is studied in photon-nuclei interactions. Accordingly, the systematics of strong nuclear amplification of gluon saturation from production of mesons

Published by the American Physical Society under the terms of the Creative Commons Attribution 4.0 International license. Further distribution of this work must maintain attribution to the author(s) and the published article's title, journal citation, and DOI. Funded by SCOAP ${ }^{3}$. in $e A$ collisions is presented in Ref. [3]. The photon-target interaction amplitude, when considering the light-cone dipole formalism [4], can be written as a convolution between the photon-meson wave functions overlap and the elementary dipole-target cross section [5]. Within the color dipole approach, one can introduce information on dynamics beyond the leading logarithmic QCD approach and computing predictions for the radially excited states is a simple task [5]. From the experimental point of view, there is intense activity on measuring the rapidity and momentum transfer distributions for coherent and incoherent processes and the theoretical models in general consider distinct dynamics for low and high mass production. On the other hand, the parton saturation framework describes both regimes in a unified way (for a recent study describing light and heavy meson photoproduction at the LHC, see, for instance, Ref. [6]).

In this work, we investigate the coherent and incoherent vector meson photoproduction in $\mathrm{Xe}+\mathrm{Xe}$ collisions at the LHC for the energy of $5.44 \mathrm{TeV}$ per nucleon. This is motivated by the test run of collisions of xenon ions recently performed at LHC, which reached a statistics of several $\mu b^{-1}$. Xenon (Xe) is around $40 \%$ lighter than that of lead $(\mathrm{Pb})$ and the QGP-like medium created in $\mathrm{Xe}+\mathrm{Xe}$ collisions would be cooler and shorter lived when compared to $\mathrm{PbPb}$ collisions. The expectations are that differences observed in the measurements from the collisions of these two nuclei could provide valuable information on the underlying physics of nuclear environment. In order to predict the cross section for $\mathrm{Xe}+\mathrm{Xe}$ collisions, we will consider the QCD color dipole approach and phenomenological models including parton saturation phenomenon. Such an analysis has been previously considered for $\mathrm{Pb}+\mathrm{Pb}$ collisions at the $\mathrm{LHC}$ with relative success. For instance, the theoretical uncertainty associated to the light meson production was investigated [7], where ALICE data 
for $\rho$ photoproduction have been described. Similar analysis was done [8] also for heavy mesons, including prediction for the incoherent cross section for $J / \psi$ and $\Upsilon$. The detailed analysis related to the radial excitations of charmonia was investigated in Refs. [9-11], where the momentum transfer distribution has been also addressed.

Our analysis here is complementary to the studies done in Ref. [12], which considers the photonuclear production of mesons $\rho$ and $\phi$ in the context of UPCs using xenon ions and the corresponding role of structure factors of Xe isotopes in xenon-based detectors of dark matter (WIMP candidates in direct dark matter searches). The theoretical approach considered was the combination of Glauber-Gribov model to describe nuclear effects (shadowing) and a model for hadronic fluctuations for the photon-nucleon cross section, which is in agreement with the experimental results for $\rho$-photoproduction at RHIC ( $\mathrm{AuAu}$ ) and $\mathrm{LHC}(\mathrm{PbPb}$ runs) energies. For the incoherent case, the authors analyze the size of the target nucleon dissociation contribution in detail. We consider it is timely and important to compare the distinct approaches presented in the literature and to estimate the size of theoretical uncertainties for the cross sections in the vector meson production.

The paper is organized as follows. In the next section, we give the main theoretical information to obtain the rapidity distribution of coherent and incoherent production of vector mesons in $\mathrm{Xe}+\mathrm{Xe}$ collisions at center-of-mass energy of $5.44 \mathrm{TeV}$. Very recent experimental analyses for these collisions are ongoing; see, for instance, Refs. [13-20]. We will demonstrate that the expected yields are enough to perform reliable cross section measurements for light mesons as $\rho^{0}$ and $\phi$. In Sec. III, we present the phenomenological calculations, discuss the main theoretical uncertainties and comparison with other approaches is performed. Finally, we summarize the main conclusions.

\section{THEORETICAL FORMALISM}

Exclusive meson photoproduction in nucleus-nucleus collisions is factorized in terms of the equivalent flux of photons of the nucleus projectile and photon-target production cross section [1]. In UPCs, there is the absence of strong interactions between the projectile particle and the target and they are characterized by impact parameter $>2$ $R_{A}$. The interaction is ultrarelativistic and purely electromagnetic and in general one uses the Weizsäcker-Williams approximation [1]. The photon energy spectrum, $d N_{\gamma}^{A} / d \omega$, which depends on the photon energy $\omega$, takes part in the expression for the rapidity distribution of vector mesons $(V)$ which is written in the following way,

$$
\begin{aligned}
\frac{d \sigma}{d y} & (A A \rightarrow A \otimes V \otimes Y) \\
& =\left[\omega \frac{d N_{\gamma}^{A}}{d \omega} \sigma(\gamma A \rightarrow V Y)+(y \rightarrow-y)\right],
\end{aligned}
$$

where $Y=A$ (coherent case) or $Y=A^{*}$ (incoherent case). The symbol $\otimes$ denotes the large rapidity gap between the produced meson and the final states nucleus.

The produced state with mass $m_{V}$ has rapidity $y \simeq$ $\ln \left(2 \omega / m_{V}\right)$ and the square of the $\gamma A$ center-of-mass energy is given by $W_{\gamma A}^{2} \simeq 2 \omega \sqrt{s}$. As one has a diffractive process, the exchanged object in the interaction carries vacuum quantum numbers (the Pomeron). Here, we consider the QCD realization of color singlet object based on the twogluon exchange and further gluon emissions. That is, the photon-Pomeron interaction will be described within the light-cone dipole frame, where the probing projectile fluctuates into a quark-antiquark pair with transverse separation $r$ (and momentum fraction $z$ ) long after the interaction, which then scatters off the hadron. The cross section for exclusive photoproduction of the meson states off a nucleon target is given by

$\sigma(\gamma p \rightarrow V p)=\frac{\left|\sum_{h, \bar{h}} \int d z d^{2} r \Psi_{h, \bar{h}}^{\gamma} \sigma_{\mathrm{dip}}(x, r) \Psi_{h, \bar{h}}^{V *}\right|^{2}}{16 \pi B_{V}}$,

where $\Psi^{\gamma}$ and $\Psi^{V}$ are the light-cone wave function of the photon and of the vector meson, respectively (we will consider in this work the following states: $V=\rho, \phi$, $J / \psi, \psi(2 S), \Upsilon(1 S), \Upsilon(2 S))$. The quark and antiquark helicities states are labeled by $h$ and $\bar{h}$, respectively. The dipoleproton cross section is denoted by $\sigma_{\text {dip }}(x, r)$ and the diffractive slope parameter by $B_{V}$. In this context, we are implicitly assuming that the proton shape is Gaussian and that the impact parameter dependence factorizes out from the dipole-nucleon scattering amplitude.

The exclusive photoproduction off nuclei for coherent and incoherent processes is computed in high energies (in the limit of large coherence length $l_{c} \gg R_{A}$ ) as follows [21],

$$
\begin{aligned}
\sigma(\gamma A \rightarrow V A)= & K^{2} \int d^{2} b \mid \sum_{h, \bar{h}} \int d z d^{2} r \Psi_{h, \bar{h}}^{\gamma} \Psi_{h, \bar{h}}^{V *} \\
& \times\left.\left[1-\exp \left(-\frac{1}{2} \sigma_{\text {dip }}(x, r) T_{A}(b)\right)\right]\right|^{2}, \\
\sigma\left(\gamma A \rightarrow V A^{*}\right)= & K^{2} \int d^{2} b \frac{T_{A}(b)}{16 \pi B_{V}} \mid \sum_{h, \bar{h}} \int d z d^{2} r \Psi_{h, \bar{h}}^{\gamma} \Psi_{h, \bar{h}}^{V *} \\
& \times\left.\sigma_{\text {dip }}(x, r) \exp \left[-\frac{1}{2} \sigma_{\text {dip }}(x, r) T_{A}(b)\right]\right|^{2},
\end{aligned}
$$

where $T_{A}(b)=\int d z \rho_{A}(b, z)$ is the nuclear thickness function. We performed the calculations with the twoparameter Fermi distribution of the nuclear density, $\left.\rho(r)=\rho_{0}\left[1+\exp \left(r-R_{A}\right) / a\right)\right]^{-1}$, with $\rho_{0}=0.148 \mathrm{fm}^{-3}$, $R_{A}=6.624 \mathrm{fm}$ and $a=0.54 \mathrm{fm}$. Concerning the incoherent case, here we will not consider neutron emission. Distinct from coherent diffraction, the nucleus is allowed 
to break up, but except for the vector meson, no new particles are produced in the reaction. We quote Ref. [22] where the multiple scattering expansion of the incoherent diffractive cross section is derived as an expansion over quasielastic scatterings of the color dipole. Our expression in Eq. (4) corresponds to the first-order term and should be the dominant contribution at small- $t$.

In the numerical evaluations, we will use boosted Gaussian wave functions and several phenomenological saturation models, which encode the main properties of the QCD parton saturation approach. The cross sections above should include both the skewedness and the real part of the amplitude corrections, and we multiply the expressions by $K^{2}=R_{g}^{2}\left(1+\beta^{2}\right)$, where $\beta=\tan \left(\pi \lambda_{\text {ef }} / 2\right)$ is the ratio of real to imaginary parts of the scattering amplitude and $R_{g}$ incorporates the off-forward correction (see, e.g., Refs. [7,8,10] for details). The effective power on energy $\lambda_{e f}$ is determined for each case. In order to take into account the threshold correction for the dipole cross section, we have multiplied them by a factor $(1-x)^{n}$ (with $n=5$ for light mesons and $n=7$ for the heavy ones).

Now, we set the parameters and phenomenological models to be considered in the next section. For the slope parameter one considers an energy dependence based on Regge phenomenology $[7,8,10]$,

$$
B_{V}=b_{e l}^{V}+2 \alpha^{\prime} \log \left(\frac{W_{\gamma A}^{2}}{W_{0}^{2}}\right)
$$

We call attention that $B_{V}$ is considered only for calculating the incoherent cross section. For the meson wave function, one takes the boosted-Gaussian model [23] because it can be applied in a systematic way for excited states. It works well for the light mesons and also for the heavier mesons, and it is given by

$$
\begin{aligned}
\psi_{\lambda, h \bar{h}}^{V}(z, r)= & \sqrt{\frac{N_{c}}{4 \pi}} \frac{\sqrt{2}}{z(1-z)}\left\{\delta_{h, \bar{h}} \delta_{\lambda, 2 h} m_{q}+i(2 h) \delta_{h,-\bar{h}} \mathrm{e}^{i \lambda \phi}\right. \\
& \left.\times\left[(1-z) \delta_{\lambda,-2 h}+z \delta_{\lambda, 2 h}\right] \partial_{r}\right\} \phi_{n S}(z, r)
\end{aligned}
$$

with $\lambda$ being the meson helicity and where $\phi_{n S}(z, r)$ is given by [24]

$$
\phi_{n S}(r, z)=\left[\sum_{k=0}^{n-1} \alpha_{n S, k} R_{n S}^{2} \hat{D}^{2 k}(r, z)\right] G_{n S}(r, z),
$$

with $\alpha_{n S, 0}=1$. The operator $\hat{D}^{2}(r, z)$ is defined by

$$
\hat{D}^{2}(r, z)=\frac{m_{f}^{2}-\left(\frac{1}{r} \partial_{r}+\partial_{r}^{2}\right)}{4 z(1-z)}-m_{f}^{2},
$$

and it acts on the following generatrix function:
TABLE I. The parameters for the diffractive $B_{V}$ slope parameter, Eq. (5), for mesons considered in the present work.

\begin{tabular}{lccc}
\hline \hline Meson & $B_{0}\left[\mathrm{GeV}^{-2}\right]$ & $\mathrm{W}_{0}[\mathrm{GeV}]$ & $\alpha^{\prime}\left[\mathrm{GeV}^{-2}\right]$ \\
\hline$\rho, \phi$ & 11.0 & 95.0 & 0.25 \\
$J / \psi$ & 4.99 & 95.0 & 0.25 \\
$\psi(2 S)$ & 4.31 & 95.0 & 0.25 \\
$\Upsilon(1 S)$ & 3.68 & 95.0 & 0.164 \\
$\Upsilon(2 S)$ & 3.61 & 95.0 & 0.164 \\
\hline \hline
\end{tabular}

$G_{n S}(r, z)=\mathcal{N}_{n S} z(1-z)$

$$
\times \exp \left(-\frac{m_{f}^{2} \mathcal{R}_{n S}^{2}}{8 z(1-z)}-\frac{2 z(1-z) r^{2}}{\mathcal{R}_{n S}^{2}}+\frac{m_{f}^{2} \mathcal{R}_{n S}^{2}}{2}\right) .
$$

For the sake of completeness, in Tables I and II are presented the parameters considered for the diffractive slope and parameters for the meson wave function, respectively.

We will use different models for the dipole scattering cross section: GBW [27], b-CGC [28] and IP-Sat [26]. The GBW model is defined by an eikonal shape for the dipole cross section,

$$
\sigma_{q \bar{q}}^{\mathrm{GBW}}(x, r)=\sigma_{0}\left(1-e^{-r^{2} Q_{s}^{2}(x) / 4}\right),
$$

where $\sigma_{0}=2 \pi R^{2}$ is a constant and $Q_{s}^{2}(x)=\left(x_{0} / x\right)^{\lambda} \mathrm{GeV}^{2}$ denotes the saturation scale. The parameters are fitted from DESY-HERA data and their values are $x_{0}=1.1 \times 10^{-4}$, $\lambda=0.287, \sigma_{0}=23.9 \mathrm{mb}$. We also consider the b-CGC model [28], based in the color glass condensate framework, in which gluon saturation effects are incorporated via an approximate solution of the Balitsky-Kovchegov equation [29]. The expression for the b-CGC model is given by

$\sigma_{q \bar{q}}^{\mathrm{BCGC}}(x, r)=2 \int d^{2} b\left\{\begin{array}{ll}\mathcal{N}_{0}\left(\frac{r Q_{s}}{2}\right)^{\gamma_{\text {eff }}(x, r)}: & r Q_{s} \leq 2 \\ 1-\mathrm{e}^{-A \ln ^{2}\left(B r Q_{s}\right)}: & r Q_{s}>2\end{array}\right.$,

where the parameter $Q_{s}$ now depends on the impact parameter,

TABLE II. Parameters of the boosted Gaussian wave function, including the quark masses.

\begin{tabular}{lcccccc}
\hline \hline Meson & $M_{V} / \mathrm{GeV}$ & $m_{f} / \mathrm{GeV}$ & $\mathcal{N}_{T}$ & $\mathcal{R}^{2} / \mathrm{GeV}^{-2}$ & $\alpha_{s_{1}}$ & $e_{f}$ \\
\hline$J / \psi[25]$ & 3.096 & 1.27 & 0.596 & 2.45 & $\cdots$ & $2 / 3$ \\
$\psi(2 \mathrm{~S})[25]$ & 3.686 & 1.27 & 0.7 & 3.72 & -0.61 & $2 / 3$ \\
$\phi[26]$ & 1.019 & 0.14 & 0.919 & 11.2 & $\cdots$ & $1 / 3$ \\
$\rho[26]$ & 0.776 & 0.14 & 0.911 & 12.9 & $\cdots$ & $1 / \sqrt{2}$ \\
$\Upsilon(1 \mathrm{~S})[24]$ & 9.46 & 4.2 & 0.481 & 0.567 & $\cdots$ & $1 / 3$ \\
$\Upsilon(2 \mathrm{~S})[24]$ & 10.023 & 4.2 & 0.624 & 0.831 & -0.555 & $1 / 3$ \\
\hline \hline
\end{tabular}



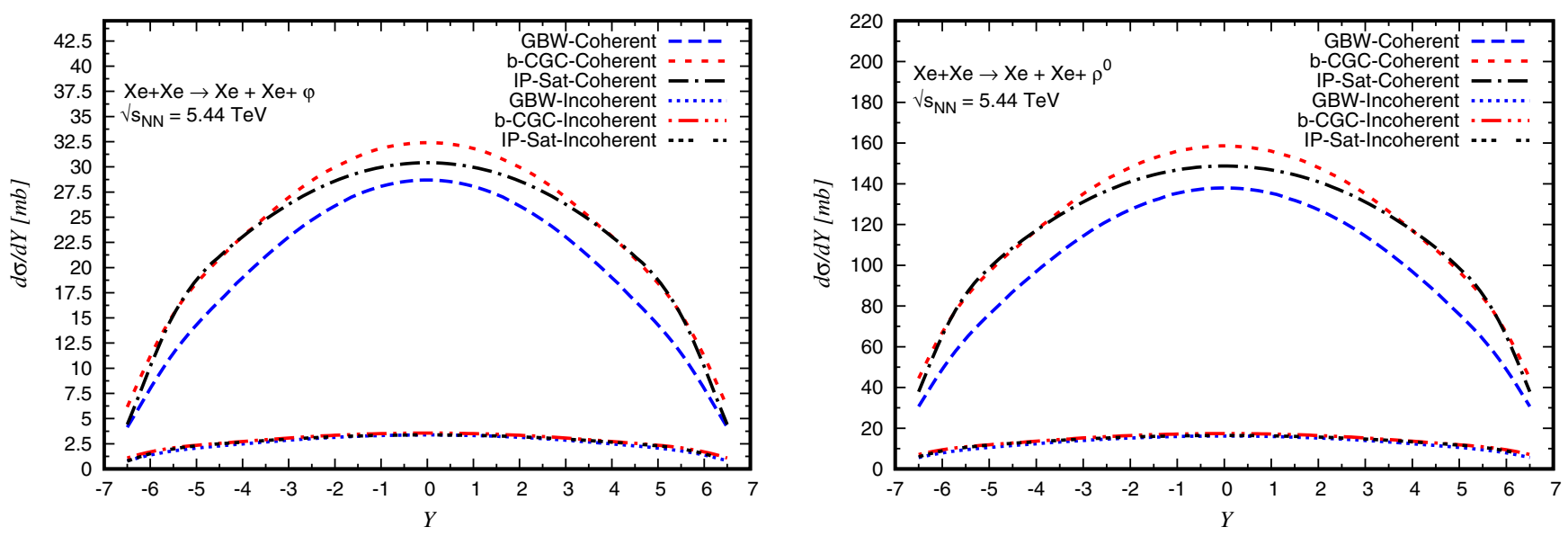

FIG. 1. Rapidity distributions for the coherent (upper curves) and incoherent (lower curves) photonuclear production of $\phi$ and $\rho$ considering GBW, b-CGC and IP-Sat color dipole models (including parton saturation phenomenon).

$$
Q_{s} \equiv Q_{s}(x, b)=\left(\frac{x_{0}}{x}\right)^{\frac{\lambda}{2}}\left[\exp \left(-\frac{b^{2}}{2 B_{\mathrm{CGC}}}\right)\right]^{\frac{1}{2 \gamma_{s}}},
$$

where $\gamma_{\mathrm{eff}}(x, r)=2\left(\gamma_{s}+(1 / \kappa \lambda \ln (1 / x)) \ln \left(2 / r Q_{s}\right)\right)$ is the effective anomalous dimension and one has the constant $\kappa=9.9$. The remaining parameters are $\mathcal{N}_{0}=0.417$, $x_{0}=5.95 \times 10^{-4}, \lambda=0.159, \quad \gamma_{s}=0.63$ and $B_{\mathrm{CGC}}=$ $5.5 \mathrm{GeV}$.

Both GBW and b-CGC models do not include DGLAP evolution in their formulation. In order to investigate the theoretical uncertainty involved in the different evolutions in saturation models, we also consider the IP-Sat model. The dipole cross section is now given by $[26,30]$,

$$
\begin{aligned}
\sigma_{\text {dip }}^{\mathrm{IP}-\mathrm{sat}}(x, r) & =2 \int d^{2} \mathbf{b} N(x, r, \mathbf{b}), \\
N(x, r, \mathbf{b}) & =1-\exp \left(-\frac{\pi^{2} r^{2} \alpha_{s}\left(\mu^{2}\right) x g\left(x, \mu^{2}\right) T(b)}{2 N_{c}}\right) .
\end{aligned}
$$

Here, $x g\left(x, \mu_{0}^{2}\right)=A_{g} x^{-\lambda_{g}}(1-x)^{5.6}$ with $\mu^{2}=\frac{4}{r^{2}}+\mu_{0}^{2}$. The profile function of the proton is considered as a gaussian, $T_{G}(b)=\frac{1}{2 \pi B_{G}} \mathrm{e}^{-\frac{b^{2}}{2 B_{G}}}$, where $B_{G}=4.0 \mathrm{GeV}^{-1}$ is obtained from the fit of $\frac{d \sigma}{d t}$ for vector meson production [26]. The remaining parameters are $\mu_{0}=1.17 \mathrm{GeV}^{2}, A_{g}=.55, \lambda_{g}=$ 0.020 and $\Lambda_{\mathrm{QCD}}=0.2 \mathrm{GeV}$.

The quantity $x g\left(x, \mu_{0}^{2}\right)$ is the input at initial scale $\mu_{0}^{2}=$ $Q_{0}^{2}$ for DGLAP evolution without quarks, since the interaction between the nucleon and color dipole occurs through the exchange of two gluons or one Pomeron. That is, the QCD evolution is given by

$\frac{\partial g\left(x, Q^{2}\right)}{\partial \log Q^{2}}=\frac{\alpha_{s}}{2 \pi}\left(\sum_{i} P_{g q} \otimes\left(q_{i}+\bar{q}_{i}\right)+P_{g g} \otimes g\right)$,

where QCDNUM [31] was used for the DGLAP evolution.
In the next section, we address the numerical calculation of coherent and incoherent cross sections in $\mathrm{Xe}+\mathrm{Xe}$ collision at the energy of $5.44 \mathrm{TeV}$ and investigate the theoretical uncertainty within the color dipole approach using the distinct implementations of the parton saturation phenomenon.

\section{RESULTS AND DISCUSSIONS}

Before calculating the cross sections, we consider the present status of experimental feasibility for the processes we are considering here. The CMS Collaboration has measured $\mathrm{Xe}+\mathrm{Xe}$ collisions with an integrated luminosity of $3.42 \mu \mathrm{b}^{-1}$ and the rapidity interval $|\eta|<1$, whereas ATLAS Collaboration has measured $3 \mu b^{-1}$ with $|\eta|<2.5$. On the other hand, ALICE Collaboration has an limited integrated luminosity of $0.34 \mu b^{-1}$ but presents an interesting analysis on $J / \psi$ production at forward rapidities, $2.5<y<4$. Given the typical efficiency for coherent vector meson production of a few percent (times the corresponding branching ratios), the expected yields should be enough to perform reliable $\rho$ or $\phi$ cross section measurements. However, the current statistics for $\mathrm{Xe}+\mathrm{Xe}$ UPCs is too low in order to investigate quarkonia events. We will present the estimates for heavy mesons for the sake of completeness having in mind it can be useful for a long time future run using xenon ions.

We show in the following the theoretical predictions for the coherent (upper curves) and incoherent (lower curves) processes for $\mathrm{Xe}+\mathrm{Xe}$ UPCs at $5.44 \mathrm{TeV}$. The analysis is focused on the rapidity distribution and theoretical uncertainty from distinct dipole cross sections. In Fig. 1, it is presented the results for photonuclear production of $\rho$ (right panel) and $\phi$ (left panel) states, taking into account the different models presented in the last section. The theoretical uncertainty is sizable being of order $6 \%-7 \%$ for the both mesons in the coherent case. There would be an additional uncertainty related to the vector meson wave 

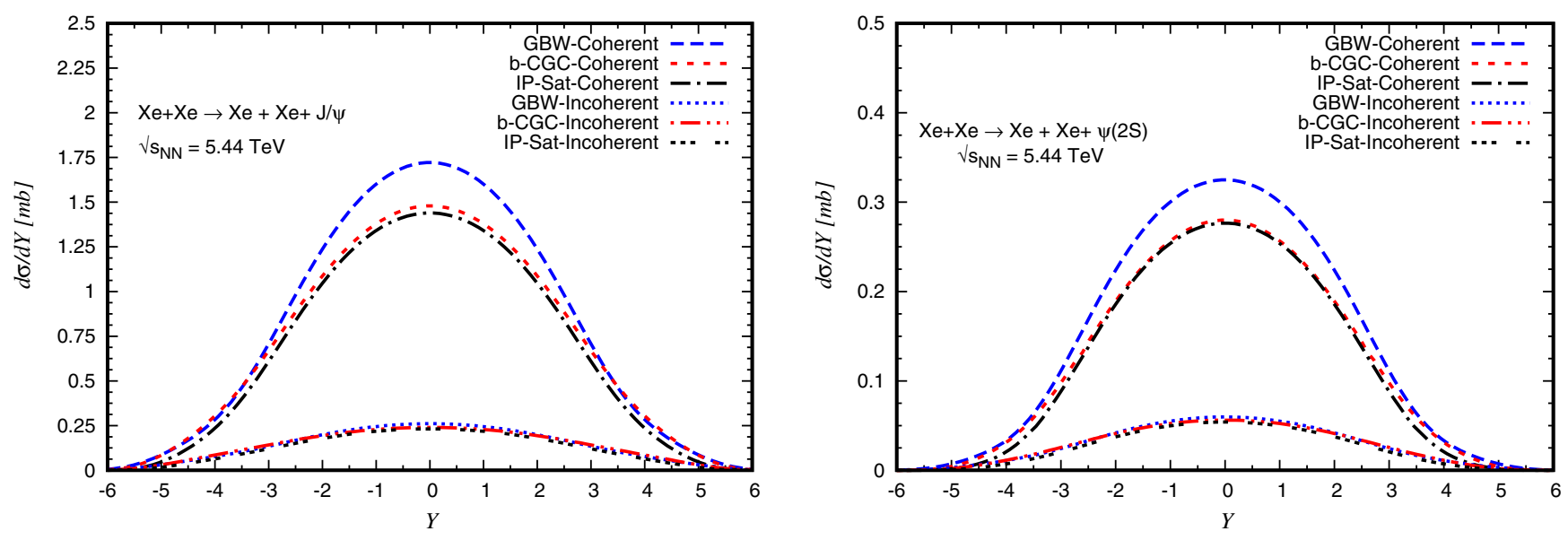

FIG. 2. Rapidity distributions for the coherent (upper curves) and incoherent (lower curves) photonuclear production of $J \psi$ and $\psi(2 S)$ for GBW, b-CGC and IP-SAT dipole models.

function (for details on the size of the related uncertainty, see, e.g., Ref. [6]). At central rapidity, one predicts explicitly $d \sigma_{\mathrm{coh}} / d y(y=0)=148.3 \pm 10.3 \mathrm{mb}$ and $d \sigma_{\mathrm{coh}} /$ $d y(y=0)=30.5 \pm 1.8 \mathrm{mb}$ for $\rho$ and $\phi$, respectively. In addition, we get $d \sigma_{\text {inc }} / d y(y=0)=8.4 \pm 0.3(1.73 \pm$ $0.05) \mathrm{mb}$ for $\rho(\phi)$ for the incoherent case, which has a smaller (around 3\%) theoretical uncertainty that the coherent cross section. The relative contribution of the coherent $\rho$ compared to the $\phi$ states is $\left(\frac{d \sigma_{\rho}}{d y}\right) /\left(\frac{d \sigma_{\phi}}{d y}\right)=4.9 \pm 0.4$ at $y=0$. In order to study the feasibility of measurements, the typical efficiency for coherent $\rho$ in ALICE [32] is around $\bar{\epsilon}=(\operatorname{Acc} \times \varepsilon)_{\rho} \sim 7 \%$ and the branching ratio $\operatorname{Br}(\rho \rightarrow \pi \pi) \simeq 1$. Thus, we would expect up to $L_{\text {int }} \times \bar{\epsilon} \times$ $\operatorname{Br} \times\left(d \sigma_{\rho} / d y\right) \sim 3.5 \times 10^{4}$ decays in the channel $\rho \rightarrow \pi \pi$ at CMS and $3.5 \times 10^{3}$ decays at ALICE per unity of rapidity.

Let us discuss the comparison to other model predictions for $\rho$ and $\phi$ photoproduction. In Ref. [12], a model based on the Glauber-Gribov approach and hadronic fluctuations for the photon-nucleon cross section, which is in agreement with the experimental results for $\rho$ photoproduction at RHIC ( $\mathrm{AuAu}$ ) and LHC ( $\mathrm{PbPb}$ runs) energies, has been considered. The authors provide predictions for low mass meson photoproduction in $\mathrm{Xe}+\mathrm{Xe}$ UPCs. For the incoherent case, they also analyze the size of target nucleon dissociation contribution in detail. That study predicts $d \sigma_{\rho} / d y(y=0) \sim 175(25) \mathrm{mb}$ for coherent (incoherent) differential cross sections, which are somewhat higher than ours. One possible source of difference is the inclusion of low-energy photoproduction related to the secondary Reggeon exchange in the $\rho-N$ interaction. Moreover, they found $d \sigma_{\phi} / d y(y=0) \sim 17.5(2.5) \mathrm{mb}$ for coherent (incoherent) $\phi$ production, showing the same trend as in the $\rho$ case. On the other hand, the dedicated STARlight MonteCarlo generator $[33,34]$ is based on the parametrization of the forward $\gamma p \rightarrow V p$ cross section, vector meson dominance (VMD) and using DESY-HERA data to fix the $\gamma p \rightarrow V p$ cross section. The cross-sections for coherent production on nuclear targets are determined using a classical Glauber calculation. In general, STARlight is consistent with experimental results for coherent and incoherent $\rho$ production in UPCs in $\mathrm{PbPb}$ mode $[32,33]$.

Also a recent study considering $\mathrm{Xe}+\mathrm{Xe}$ collisions has been done in Ref. [35], where photoproduction of vector mesons off proton and off nuclear targets using color dipole model in an approach that includes hot spots [36]. The hot spots have position in the impact-parameter plane changing event by event, and their number depends on the energy of $\gamma p$ system. The authors have found for the cross section at midrapidity for $\rho$ production the following values: $167.5 \pm$ $7.5 \mathrm{mb}$ (coherent $\mathrm{Xe}+\mathrm{Xe}$ ) and $8.15 \pm 1.65 \mathrm{mb}$ (incoherent $\mathrm{Xe}+\mathrm{Xe}$ ). The lower bounds correspond to the case considering nucleons with hot spots. The results are in agreement with our predictions, mostly for the incoherent cross section.

We now focus on the photoproduction of heavy mesons and their excited states, which it is presented in Figs. 2 and 3. The rapidity distributions for $J / \psi$ (left panel) and $\psi(2 S)$ (right panel) are shown in Fig. 2 using the same notation as the previous figure. As already mentioned, the incoherent cross sections are represented by the lower curves. One obtains $d \sigma_{\mathrm{coh}} / d y(y=0)=1.58 \pm 0.14 \mathrm{mb}(0.30 \pm 0.02 \mathrm{mb})$ for $J / \psi(\psi(2 S))$, with the ratio being $\sigma(\psi(2 S)) / \sigma(J / \psi)$ of around 0.2 . The theoretical uncertainty seems to be smaller than for the light mesons. The incoherent cross section is typically $20 \%$ of the coherent one. Notice that the ratio $\sigma_{\text {inc }} / \sigma_{\text {coh }}$ provides further constraints on the treatment of the nuclear modifications implemented in the different models. Now the current statistics is quite low, given an efficiency for coherent $J / \psi$ in CMS ( $\bar{\epsilon}$ simeq6\%) and ALICE [37] to be $\bar{\epsilon}=(\operatorname{Acc} \times \varepsilon)_{J / \psi} \sim 16 \%$ and the branching ratio $\operatorname{Br}\left(J / \psi \rightarrow \ell^{+} \ell^{-}\right) \simeq 0.06$. One gets $L_{\text {int }} \times \bar{\epsilon} \times \mathrm{Br} \times$ $\left(d \sigma_{\psi} / d y\right) \sim 42$ decays in mode $J / \psi \rightarrow \mu^{+} \mu^{-}$at CMS and 5 decays at ALICE per unity of rapidity. Finally, the rapidity 

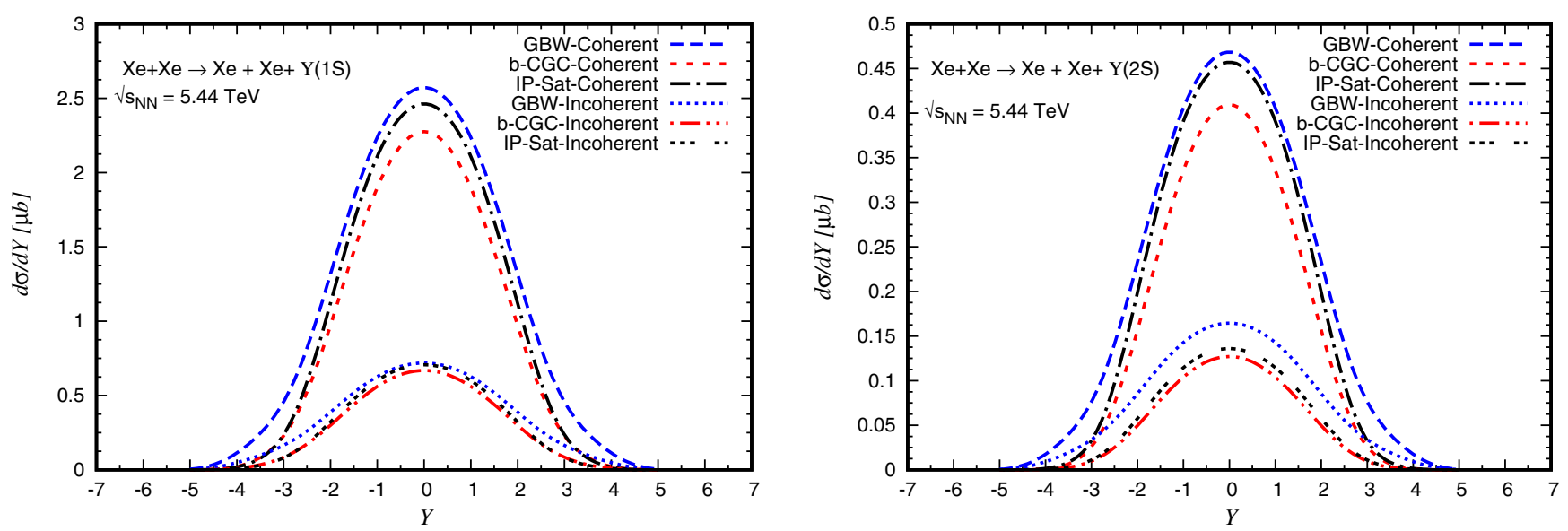

FIG. 3. Rapidity distributions for the coherent (upper curves) and incoherent (lower curves) photonuclear production of (1S) and $\Upsilon(2 \mathrm{~S})$ for GBW, b-CGC and IP-SAT dipole models.

distributions for $\Upsilon(1 S)$ (left panel) and $\Upsilon(2 S)$ (right panel) are shown in Fig. 3 for completeness.

\section{SUMMARY}

We presented the predictions of rapidity distribution for the exclusive vector meson photoproduction for the LHC run using $\mathrm{Xe}+\mathrm{Xe}$ collisions at the energy $5.44 \mathrm{TeV}$. Predictions for the coherent (without nucleus breack up) and incoherent cross sections are presented using the color dipole approach and Glauber-Gribov treatment of nuclear shadowing. The main focus is on the gluon saturation approach, where the main quantity is the nuclear saturation scale which is the typical momentum scale for the problem. It is considered a consistent formalism, where the wave function of bound states and their radial excitations are theoretically well constrained (boosted Gaussian wave function). We show that the expected yields are enough to perform reliable cross section measurements for light mesons as $\rho^{0}$ and $\phi$. Namely, we predict up to $3.5 \times 10^{4}$ decays in the mode $\rho \rightarrow \pi \pi$ at CMS and $3.5 \times 10^{3}$ decays at ALICE per unity of rapidity. For heaviest mesons the current statistics is quite low, but the first Xe-Xe collisions have demonstrated the potential of lighter species as a path to higher hadronic luminosity.

\section{ACKNOWLEDGMENTS}

This work was partially financed by the Brazilian funding agency Conselho Nacional de Desenvolvimento Científico e Tecnológico (CNPq).
[1] G. Baur, K. Hencken, D. Trautmann, S. Sadovsky, and Y. Kharlov, Phys. Rep. 364, 359 (2002); C. A. Bertulani, S. R. Klein, and J. Nystrand, Annu. Rev. Nucl. Part. Sci. 55, 271 (2005).

[2] F. G. Ben, M. V. T. Machado, and W. K. Sauter, Phys. Rev. D 96, 054015 (2017).

[3] H. Mäntysaari and R. Venugopalan, Phys. Lett. B 781, 664 (2018).

[4] N. N. Nikolaev and B. G. Zakharov, Phys. Lett. B 332, 184 (1994); Z. Phys. C 64, 631 (1994).

[5] J. Nemchik, N. N. Nikolaev, E. Predazzi, and B. G. Zakharov, Phys. Lett. B 374, 199 (1996).

[6] V.P. Gonçalves, M. V. T. Machado, B.D. Moreira, F. S. Navarra, and G. S. dos Santos, Phys. Rev. D 96, 094027 (2017).

[7] G. S. dos Santos and M. V. T. Machado, Phys. Rev. C 91, 025203 (2015).
[8] G. S. dos Santos and M. V. T. Machado, J. Phys. G 42, 105001 (2015).

[9] M. B. G. Ducati, F. Kopp, and M. V. T. Machado, Phys. Rev. D 96, 054001 (2017).

[10] M. B. G. Ducati, F. Kopp, M. V. T. Machado, and S. Martins, Phys. Rev. D 94, 094023 (2016).

[11] M. B. G. Ducati, M. T. Griep, and M. V. T. Machado, Phys. Rev. C 88, 014910 (2013).

[12] V. Guzey, E. Kryshen, and M. Zhalov, Phys. Lett. B 782, 251 (2018).

[13] S. Acharya et al. (ALICE Collaboration), arXiv:1805.04383.

[14] S. Acharya et al. (ALICE Collaboration), arXiv:1805.04399.

[15] S. Acharya et al. (ALICE Collaboration), arXiv:1805.01832.

[16] CMS Collaboration, Report No. CMS-PAS-HIN-18-001.

[17] CMS Collaboration, Report No. CMS-PAS-HIN-18-004.

[18] CMS Collaboration, Report No. CMS-PAS-HIN-17-006. 
[19] ATLAS Collaboration, Report No. ATLAS-CONF-2018011.

[20] ATLAS Collaboration, Report No. TLAS-CONF-2018-007.

[21] B. Z. Kopeliovich and B. G. Zakharov, Phys. Rev. D 44, 3466 (1991); Yu. P. Ivanov, J. Huefner, B. Z. Kopeliovich, and A. V. Tarasov, AIP Conf. Proc. 660, 283 (2003).

[22] A. Łuszczak and W. Schäfer, Phys. Rev. C 97, 024903 (2018).

[23] J. Nemchik, N. N. Nikolaev, E. Predazzi, and B. G. Zakharov, Z. Phys. C 75, 71 (1997).

[24] B. E. Cox, J. R. Forshaw, and R. Sandapen, J. High Energy Phys. 06 (2009) 034.

[25] N. Armesto and A. H. Rezaeian, Phys. Rev. D 90, 054003 (2014).

[26] H. Kowalski, L. Motyka, and G. Watt, Phys. Rev. D 74, 074016 (2006).

[27] K. Golec-Biernat and M. Wüsthoff, Phys. Rev. D 59, 014017 (1998).

[28] E. Iancu, K. Itakura, and S. Munier, Phys. Lett. B 590, 199 (2004); G. Soyez, Phys. Lett. B 655, 32 (2007).
[29] F. Gelis, E. Iancu, J. Jalilian-Marian, and R. Venugopalan, Annu. Rev. Nucl. Part. Sci. 60, 463 (2010); H. Weigert, Prog. Part. Nucl. Phys. 55, 461 (2005); J. Jalilian-Marian and Y. V. Kovchegov, Prog. Part. Nucl. Phys. 56, 104 (2006).

[30] A. H. Rezaeian and I. Schmidt, Phys. Rev. D 88, 074016 (2013).

[31] M. Botje, Comput. Phys. Commun. 182, 490 (2011); arXiv:1602.08383.

[32] J. Adam et al. (ALICE Collaboration), J. High Energy Phys. 09 (2015) 095.

[33] S. R. Klein, J. Nystrand, J. Seger, Y. Gorbunov, and J. Butterworth, Comput. Phys. Commun. 212, 258 (2017).

[34] S. Klein and J. Nystrand, Phys. Rev. C 60, 014903 (1999).

[35] J. Cepila, J. G. Contreras, M. Krelina, and J. D. Tapia Takaki, arXiv:1804.05508.

[36] J. Cepila, J. G. Contreras, and M. Krelina, Phys. Rev. C 97, 024901 (2018).

[37] V. Khachatryan et al. (CMS Collaboration), Phys. Lett. B 772, 489 (2017). 